\title{
ANÁLISE DOCUMENTAL SOBRE OS SUICÍDIOS OCORRIDOS NA REGIÃO DE JUNDIAÍ ENTRE 2004 E 2014
}

DOCUMENTARY ANALYSIS OF THE SUICIDES IN THE JUNDIAÍ REGION BETWEEN 2004 AND 2014

DOI: 10.22199/S07187475.2016.0002.00006

Recibido: 21 de Junio de 2016 | Aceptado: 25 de Agosto de 2016

\section{FELIPE AUGUSTO CUNHA 1 ; MAKILIM NUNES BAPTISTA 1 ; LUCAS DE FRANCISCO CARVALHO 1}

1. UNIVERSIDADE SÃO FRANCISCO, Itatiba, Brasil

\begin{abstract}
RESUMO
INTRODUÇÃO: 0 suicídio é considerado um problema de saúde pública no Brasil, por isso a importância de realizar estudos documentais para obter dados referente a uma determinada região, sobre os casos de suicídio. OBJETIVO: Obter dados sociodemográficos e números de mortes por suicídio relacionados na região de Jundiaí. MÉTODO: Para esse estudo foram utilizados laudos de suicídio (total 175) do IML de uma cidade do interior do estado de São Paulo que também é responsável por mais nove cidades pertencentes da região de Jundiaí, entre os anos de 2004 e 2014 . Foram analisados dados sociodemográficos como ano, sexo, idade, localidade (urbana/rural), estado civil e o método utilizado na morte. RESULTADOS: 0 suicídio foi mais prevalente no sexo masculino (82,0\%) no meio urbano $(87,4 \%)$, seguido da faixa etária mais atingida de 20 a 40 anos para ambos os sexos. 0 estado civil com maior prevalência de suicídio foram os solteiros $(43,4 \%)$ e 0 método mais utilizado foi 0 enforcamento, tanto para os homens $(80,9 \%)$ quanto para as mulheres $(60,7 \%)$. Dentre os 11 anos pesquisados, os laudos que tiveram o maior número de morte por suicídios foram correspondentes aos anos de 2014, com 44 laudos, e 0 ano de 2013 com 34 laudos. CONCLUSÕES: 0 s resultados indicaram que os dados encontrados foram ao encontro da literatura, mostrando que 0 perfil sociodemográfico aferido na pesquisa era esperado. Portanto verifica-se a importância de estudos com esse cunho de prevalência para criar meios de políticas públicas pautadas na prevenção do suicídio no Brasil.
\end{abstract}

PALAVRAS-CHAVE: Análise documental, suicídio, mortalidade.

\section{ABSTRACT}

INTRODUCTION: Nowadays, suicide has been labeled as a public health problem in Brazil. Therefore, documentary research that brings light on this issue in several regions is not only relevant, but also necessary. OBJECTIVE: To obtain sociodemographic data and suicide statistics in the Jundiai area. METHOD: 175 suicide reports ranging from 2004 to 2014, from the Instituto Médico Legal in a city from São Paulo State in the Judiani area were consulted. Data such as year, sex, age, location (urban or rural), marital status and modus operandi from the victims were analyzed. RESULTS: Suicide is more frequent in males $(82,00 \%)$, and in the city $(87,4 \%)$. Age in suicide cases ranges from 20 through to 40 years old for both sexes. Suicide is more frequent in single people $(43,4 \%)$. Hanging is the most common method for males $(80,9 \%)$ as well as for females $(60,7 \%)$. The highest numbers of suicide cases were reported in 2014 (44 cases) and 2013 (34 cases) respectively. CONCLUSIONS: These results and the average profile of a person committing suicide match previous research and the literature in the field. This study emphasizes the importance of this type of research which serves as scenario to promote prevention of suicide in Brazil.

KEY WORDS: Burnout, MBI, Peru, religious, stress, validation.

Para más información, puede comunicarse con el autor al siguiente e-mail: flpcunha@terra.com.br, makilim01@gmail.com, lucas@labape.com.br 


\section{INTRODUÇÃO}

A Organização Mundial da Saúde (OMS) publicou um manual de prevenção de suicídio, em 2015, contendo várias informações sobre os últimos dados estatísticos das mortes ocorridas por suicídio, também denominado autoextermínio; etimologicamente a palavra foi extraída do latim e significa sui (si mesmo) e caedes (ação de matar) (Correa \& Barrero, 2006). 0 autoextermínio pode ser compreendido como uma ação deliberada, iniciada e finalizada pelo próprio indivíduo com consciência e conhecimento do resultado final de seu ato, levando a morte $\mathrm{e}$ os números de óbitos de suicídio pelo mundo é de aproximadamente um milhão (OMS, 2014). Os adolescentes com idade entre 15 e 19 anos, apareceram no estudo como um grupo de alto risco, juntamente da população correspondente da terceira idade (Moreira \& Bastos, 2015).

Alguns países do mundo apresentam uma taxa média de suicídios maior do que média global (11,4 por 100 mil), entre esses países encontram-se Lituânia $(38,6)$, Japão $(23,7)$ e Suécia $(12,8)$; enquanto Kuwait $(2,0)$ e África do Sul $(1,0)$ tem as médias mundiais mais baixas. Já na América do Sul, Uruguai $(15,5)$, é o pais com o índice maior de mortes por suicídios, seguido da Bolívia (12,2), Chile $(12,2)$, Argentina $(8,1)$, Brasil (5,1) e Venezuela (4,2) (Botega, 2015; OMS, 2014).

Algumas interpretações sugerem que 0 levantamento sobre suicídio deve ser ponderado pelo gênero (OMS, 2014), de modo que os homens cometem mais suicídio do que as mulheres. No ano de 2012, aproximadamente 9.000 homens se mataram no Brasil, 0 equivalente a 9,0 mortes a cada 100 mil habitantes, ao passo que no ano de 2000 a taxa foi de 8,7 mortes, apresentando um aumento de 0,3\%. Em relação as mulheres, o número de mortes foi de 2.623 casos em uma taxa de 2,5/100 mil habitantes, apresentando uma diferença expressiva entre os sexos. No total, 11.821 pessoas se mataram no Brasil no ano de 2012.

Ainda comentando sobre números do Brasil, a faixa etária mais comum entre as mortes por suicídios encontra-se nas pessoas com idade superior a 60 anos, havendo um aumento de morte em pessoas com 80 anos ou mais, portanto, a maior incidência de mortes ocorre na população dos idosos; o crescimento apontou um aumento de $154 \%$ em indivíduos que tem idade superior a 80 anos, entre os anos de 1996 e 2012 (DATASUS, 2014).

Algumas das causas encontradas que estão associadas ao suicídio comumente são os transtornos psiquiátricos, sendo a mais frequente a depressão e também 0 uso abusivo de álcool e outras drogas (Dutra, 2012). A Associação Brasileira de Psiquiatria (2014) comenta em seu manual de prevenção do suicídio que existem alguns fatores de risco que contribuem para o autoextermínio, que são os transtornos mentais, as questões sociodemográficas, psicológicas e condições clínicas incapacitantes. Os transtornos mentais mais comuns nos casos de suicídio são os transtornos de humor dando ênfase para a depressão, transtornos de personalidade, principalmente a esquizofrenia e transtornos de ansiedade (Hawton, Comabella, Haw \& Saunders, 2013; Miret, Ayuso-Mateos, Sanchez-Moreno \& Vieta, 2013).

Em especial nos idosos, existem outros agravantes que suscitam a ideação suicida, como por exemplo a gênese de algumas doenças crônicas incuráveis, a perda da representação social exercida pelo trabalho, - luto de pessoas próximas e também a diminuição de energia vital, incapacitando a realização de atividades, que antes proporcionavam prazer (OMS, 2014).

Outro fator diretamente ligado às mortes são os métodos mais utilizados no momento do autoextermínio. Botega (2014) comentou 
que os meios usados no suicídio mudam a partir de interferências da cultura no indivíduo; outros fatores que também influenciam o estilo do método adotado para a morte no suicídio, tem ligação com 0 gênero e a faixa etária. Na Inglaterra, por exemplo, o enforcamento e a intoxicação por gases são mais usados, já nos Estados Unidos, a arma de fogo, enquanto que no Sri Lanka o envenenamento por pesticidas. Berlote (2012) explica que no Brasil os principais meios utilizados são 0 enforcamento (47\%), armas de fogo (19\%) e envenenamento (14\%). Em relação ao sexo, os homens usam mais do enforcamento (58\%), arma de fogo (17\%) e envenenamento por pesticidas (5\%). As mulheres usam mais o enforcamento (49\%), fumaça/fogo $(9 \%)$, precipitação de altura $(6 \%)$, arma de fogo $(6 \%)$ e envenenamento $(5 \%)$.

Além dos fatores de riscos mais conhecidos, como os transtornos psiquiátricos sendo os principais, os depressivos, transtorno bipolar e o uso abusivo de substâncias químicas, existe também a importância de especular o perfil sociodemográfico das pessoas que cometem atos suicidas, focando em políticas públicas para favorecer a prevenção desse mal que cresce cada vez mais, não só no Brasil, mas no mundo (Lovisi, Santos, Legay, Abelha \& Valencia, 2009; Baptista, 2004). Assim, o objetivo desta pesquisa é apresentar e analisar de forma comparativa com outros dados algumas características demográficas e sociais referentes aos suicidas de uma região específica do interior de São Paulo por meio de laudos do Instituto Médico Legal (IML) entre os anos de 2004 e 2014, incluindo os principais métodos para cometer suicídio na região. Os resultados da pesquisa foram comparados posteriormente com outros dados.

A metodologia da pesquisa envolve duas etapas. Sendo a primeira, uma revisão da literatura com 0 intuito de explorar sobre a prevalência do suicídio no Brasil, utilizando duas bases de dados para executar a pesquisa, a Scientific Electronic Library Online (SciELO) e o Periódicos eletrônicos em Psicologia (PePSIC), em Fevereiro de 2016. As buscas nas bases foram feitas utilizando as seguintes palavras-chave: suicídio, prevalência, epidemiologia e incidência; todas acompanhadas com a palavra "suicídio". Os critérios de inclusão foram 0 estudo ser brasileiro, artigo completo e ser realizado nos últimos 11 anos, entre 2005 e 2015.

A segunda etapa foi usada para a seleção dos artigos da pesquisa nas bases de dados, o SciELO a primeira base pesquisada, encontrando 33 artigos com 0 uso das palavras-chave: suicídio e prevalência, desse total, somente 2 artigos foram utilizados na pesquisa. Com as palavras-chave: suicídio e epidemiologia, 11 artigos foram encontrados, restando 3 , e por fim, utilizando as palavras-chave suicídio e incidência, os artigos encontrados foram 13, sendo utilizando somente 2. 0 banco de dados PePSIC teve um resultado ínfimo comparado com o SciELO, utilizando todas as palavras-chave, somente foram encontrados 4 artigos, sendo utilizado apenas 1 , que já havia sido encontado no SciELO.

De todo os artigos pesquisados, somente foram encontrados 61 , sendo utilizado as palavras-chave supracitado, no total, somente 11 foram utilizados, os demais artigos foram excluídos porque fugiam do tema da pesquisa, como por exemplo, estudos que avaliavam suicídios ocorridos em gestantes ou mesmo suicídios ocorridos por agrotóxicos em determinadas plantações, além de artigos replicados. Após as pesquisas e análises, oito artigos foram mantidos e descritos na Tabela 1 , classificados por autores e anos da publicação, contendo os dados sociodemográficos mais prevalentes. 
TABELA 1.

Artigos sobre os suicídios.

\begin{tabular}{|c|c|c|c|c|c|c|}
\hline $\begin{array}{l}\text { Autor e Ano } \\
\text { (Publicação) }\end{array}$ & $\begin{array}{l}\text { Sexo } \\
(\%)\end{array}$ & Idade & $\begin{array}{l}\text { Estado/ } \\
\text { Região }\end{array}$ & $\begin{array}{l}\text { Estado Civil } \\
(\%)\end{array}$ & $\begin{array}{l}\text { Método } \\
(\%)\end{array}$ & $\begin{array}{l}\text { Média de mortes } \\
(100 \mathrm{mil} / \mathrm{hab})\end{array}$ \\
\hline $\begin{array}{c}\text { Baptista, Carneiro, } \\
\text { Gomes \& Cardoso } \\
\text { (2012) }\end{array}$ & $\begin{array}{l}\text { Homem } \\
(84,9)\end{array}$ & $20-40$ & SP & $\begin{array}{c}\text { Solteiro } \\
(38,2) \\
\text { Casado } \\
(27,5)\end{array}$ & $\begin{array}{c}\text { Enforcamento } \\
(62,5) \\
\text { Arma de Fogo } \\
(18,8)\end{array}$ & 5,4 \\
\hline $\begin{array}{c}\text { Bezerra,Werneck, } \\
\text { Almeida, Oliveira \& } \\
\text { Magalhães } \\
\text { (2012) }\end{array}$ & $\begin{array}{c}\text { Homem } \\
(64,4)\end{array}$ & $40-49$ & RJ & - & -- & 5,6 \\
\hline $\begin{array}{c}\text { Macente \& } \\
\text { Zandonade } \\
\text { (2012 (Viana, } \\
\text { Zenkner, Sakae, \& } \\
\text { Escobar, 2008)) }\end{array}$ & $\begin{array}{l}\text { Homem } \\
(76,0)\end{array}$ & $50-59$ & ES & $\begin{array}{l}\text { Solteiro } \\
(38,16) \\
\text { Casado } \\
(38,82)\end{array}$ & $\begin{array}{c}\text { Enforcamento } \\
(45,39) \\
\text { Envenenamento } \\
(11,84)\end{array}$ & 4,5 \\
\hline $\begin{array}{c}\text { Lovisi, } \\
\text { Santos,Legay, } \\
\text { Abelha \& Valencia } \\
(2009)\end{array}$ & $\begin{array}{l}\text { Homem } \\
(70,3)\end{array}$ & $59-70$ & SP & - - & $\begin{array}{l}\text { Enforcamento } \\
\quad(48,8) \\
\text { Arma de Fogo } \\
\quad(16,5)\end{array}$ & 6,8 \\
\hline $\begin{array}{l}\text { Viana, Zenkner, } \\
\text { Sakae \& Escobar } \\
(2008)\end{array}$ & $\begin{array}{l}\text { Homem } \\
(79,6)\end{array}$ & $40-45$ & RS & $\begin{array}{c}\text { Solteiro } \\
(26,5) \\
\text { Casado } \\
(28,6)\end{array}$ & $\begin{array}{c}\text { Enforcamento } \\
(68,4) \\
\text { Arma de Fogo } \\
(12,2)\end{array}$ & 7,9 \\
\hline $\begin{array}{l}\text { Parente, Soares, } \\
\text { Araújo, Cavalcante } \\
\text { \& Monteiro } \\
(2007)\end{array}$ & $\begin{array}{l}\text { Homem } \\
(71,3)\end{array}$ & $20-29$ & NO & $\begin{array}{c}\text { Solteiro } \\
(67,6) \\
\text { Casado } \\
(45,7)\end{array}$ & $\begin{array}{c}\text { Enforcamento } \\
(66,0) \\
\text { Arma de Fogo } \\
(13,1)\end{array}$ & 2,4 a 3,1 \\
\hline $\begin{array}{c}\text { Pordeus, } \\
\text { Cavalcanti, Vieira, } \\
\text { Coriolano, Et al. } \\
(2007)\end{array}$ & $\begin{array}{c}\text { Homem } \\
(63,2)\end{array}$ & $40-49$ & CE & - - & $\begin{array}{c}\text { Enforcamento } \\
(32,6) \\
\text { Envenenamento } \\
(10,2)\end{array}$ & 3,8 \\
\hline $\begin{array}{c}\text { Baptista \& Borges } \\
(2005)\end{array}$ & $\begin{array}{l}\text { Homem } \\
(82,8)\end{array}$ & $30-40$ & SP & $\begin{array}{c}\text { Solteiro } \\
(18,5) \\
\text { Casado } \\
(21,4)\end{array}$ & $\begin{array}{c}\text { Enforcamento } \\
(47,6) \\
\text { Arma de Fogo } \\
(13,8)\end{array}$ & 4,9 \\
\hline
\end{tabular}

\section{MÉTODO}

\section{Fonte de dados}

Este estudo caracteriza-se como uma pesquisa documental realizada no Instituto Médico Legal de Jundiaí (IML) situado em um município do estado de São Paulo no ano de 2015, por meio do levantamento de 175 laudos de suicídio entre os anos de 2004 e 2014, sendo esses laudos arquivados em fichas de prontuários e em sistemas informatizados. Algumas dificuldades foram percebidas durante a coleta do material, alguns prontuários, não apresentaram algumas das descrições 
necessárias, como por exemplo, estado civil e método utilizado para provocar o suicídio, por isso foram excluídos cinco prontuários, que não continham as informações necessárias para este estudo. Os anos de 2014, 2013 e 2012 foram pesquisados nos sistemas informatizados, por meio de prontuários digitalizados arquivados em um sistema especifico. Os dados coletados e referem à: sexo, idade, estado civil, zona rural/urbana, método utilizado no suicídio e ano. Na Tabela 1 foram explorados os dados sociodemográgicos das pesquisas, divididos por ano de publicação, sexo, idade, estado/região, estado civil, método e média de mortes por cada 100 mil habitantes. E também foi utilizada no presente estudo para elaboração das hipóteses, de modo que, (H1) os homens cometem mais suicídios; (H2) os indivíduos com mais de 60 anos cometem mais suicídios; (H3) os métodos de suicídios mais utilizados devem ser enforcamento e uso da arma de fogo.

\section{Procedimentos}

Após autorização do médico ${ }^{1}$ responsável do IML de Jundiaí que funciona por meio de um acordo entre o Governo do Estado e a SETEC (Secretaria de Educação Profissional e Tecnológica). 0 Instituto Médico Legal é responsável por dados estatísticos sobre perícias em medicina legal de nove cidades da região: Campo Limpo Paulista, Várzea Paulista, Louveira, Vinhedo, Itupeva, Jarinu, Cabreúva, Cajamar e Morungaba. Para considerar os números de suicídios entre os anos de 2004 a 2014, foi utilizada a décima revisão da Classificação Internacional de Doenças (CID-10) (OMS, 2000), sendo as categorias listadas nesse manual, incluindo as categorias sobre as mortes por suicídio.

A aprovação do projeto de pesquisa pelo Comitê de Ética em Pesquisa da Universidade São Francisco, a coleta

1. Agradecimento ao Dr. Eugênio Carlos Grohmann pela autorização da coleta de dados. ocorreu em um prazo de 3 meses, por meio da leitura das informações dos laudos arquivados em fichas e no sistema informatizado, em uma sala no próprio local. Esses laudos foram selecionados por uma profissional da instituição responsável pelo arquivo desses prontuários, a mesma foi treinada pelo pesquisador com o intuito de sanar algumas dúvidas durante a pesquisa, por tanto a profissional contava com um material de auxilio durante a coleta de dados, que no caso foi tabelas com as características necessárias que a funcionária deveria selecionar, levando em consideração apenas os prontuários que apresentaram óbitos por suicídio. A coleta de dados somente não foi realizada pelos pesquisadores porque a instituição não permitiu manuseio dos arquivos por indivíduos que não pertenciam ao Instituo Médico Legal.

Foram avaliados 175 prontuários pela funcionária da instituição orientada pelos pesquisadores, contendo vários tipos de métodos utilizados no ato de suicídio. Os dados sociodemográficos e os métodos utilizados no ato do suicídio foram analisados por meio do programa SPSS (Statistical Package for the Social Sciences - versão 21.0). Inicialmente, foi construída uma tabela com os dados da amostra, sendo esses separados por categorias classificadas em: ano do suicídio, sexo, idade, estado civil e método utilizado. Posteriormente, foram realizadas análises descritivas para verificar a distribuição da amostra (número de participantes, idade, média e desvio padrão).

\section{RESULTADOS}

No período pesquisado, foi encontrada pelo menos uma ocorrência a cada ano (entre 2004 e 2014), todas no perímetro urbano da região de Jundiaí. A idade média da amostra foi de 43,1 anos e o DP= 19,29 anos sendo que as mulheres possuíam maior média de idade $(M=43,4$; entre $18 \mathrm{e}$ 77 anos), DP= 18,33; quando comparadas 
aos homens ( $M=43,0$; entre 8 e 8 anos), $\mathrm{DP}=19,52$. A maior prevalência de suicídiosocorreu na faixa etária de 19 e 35 anos $(48,4 \%)$ seguida pela terceira idade, que foi as pessoas que apresentaram suicídios a partir dos 67 anos $(20,4 \%)$ em ambos os sexos. Dos 175 casos pesquisados, os homens apresentaram maior número de ocorrências (147), totalizando $84 \%$ da amostra.

Entre os anos pesquisados apareceram artigos de 2005 a 2012, o sexo masculino foi o mais prevalente, a faixa etária variou entre 20 e 70 anos, mostrando um número maior de suicídios na faixa dos 40 anos. 0 estado de São Paulo apareceu na maioria dos estudos, o estado civil mais comum entre os artigos foi casado e solteiros, sendo os casados com maior índice de morte e, por fim, o método de suicídio mais averiguado nas pesquisas foi 0 enforcamento e em segundo lugar o uso da arma de fogo.
Quanto ao estado civil, o suicídio foi mais frequente nos solteiros ( $N=76 / 43,4 \%$ ), e em segundo lugar ficaram os casados ( $N=66 / 37,7 \%)$; os separados, viúvos e divorciados ficaram no menor grupo, totalizando 18,9\% da amostra. Em relação a localidade onde foram executados os atos de suicídio, a zona urbana apareceu com maior frequência, com 152 casos (87,4\%).

No que se refere aos métodos mais utilizados, em relação aos homens foram: enforcamento 119 dos casos $(80,9 \%)$ e arma de fogo 12 dos casos (10,9\%), enquanto os métodos mais utilizados pelas mulheres foram 0 enforcamento 17 dos casos $(60,7 \%)$, seguido de intoxicação medicamentosa, sete dos casos $(25,0 \%)$. Em relação aos anos pesquisados, 2014 foi 0 de maior ocorrência, com 44 suicídios, correspondendo a $25,1 \%$ dos 175 laudos analisados, seguido pelo ano de $2013 \mathrm{com}$ 34 ocorrências, $19,4 \%$. Os outros 9 anos que fizeram parte da pesquisa entre $2004 \mathrm{e}$ 2012, corresponderam no total $97(44,5 \%)$ suicídios. A Tabela 2 sintetiza as informações sobre os suicídios ocorridos nos anos de 2013 e 2014.

TABELA 2.

Total dos anos pesquisados de suicídio.

\begin{tabular}{cccccc}
\hline $\begin{array}{c}\text { Ano Suicídio e } \\
\text { No de laudos }\end{array}$ & $\begin{array}{c}\text { Sexo } \\
(\%)\end{array}$ & Idade & $\begin{array}{c}\text { Estado Civil } \\
(\%)\end{array}$ & $\begin{array}{c}\text { Método } \\
(\%)\end{array}$ & $\begin{array}{c}\text { Média de } \\
\text { mortes } \\
(100 \mathrm{mil} / \mathrm{hab})\end{array}$ \\
\hline $\begin{array}{c}\text { Homem } \\
(44)\end{array}$ & $20-40$ & $\begin{array}{c}\text { Solteiro }(54,5) \\
\text { Casado } \\
(18,2)\end{array}$ & $\begin{array}{c}\text { Enforcamento } \\
(100,0)\end{array}$ & 3,7 \\
\hline 2013 & $\begin{array}{c}\text { Homem } \\
(91,2)\end{array}$ & $20-40$ & $\begin{array}{c}\text { Solteiro }(58,2) \\
\text { Casado } \\
(41,8)\end{array}$ & $\begin{array}{c}\text { Enforcamento } \\
(82,4) \\
\text { Arma de Fogo } \\
(17,6)\end{array}$ & 4,9 \\
\hline $\begin{array}{c}\text { Total/Média } \\
(175)\end{array}$ & $\begin{array}{c}\text { Homem } \\
(70,6)\end{array}$ & 43,2 & $\begin{array}{c}\text { Solteiro } \\
(30,9) \\
\text { Casado } \\
(30,4)\end{array}$ & $\begin{array}{c}\text { Enforcamento } \\
(70,1)\end{array}$ \\
& & & Arma de Fogo \\
$(10,0)$ & 5,6 \\
\hline
\end{tabular}


$\mathrm{Na}$ Tabela 2 estão expostos dois anos que tiveram mais prontuários dentre os 11 anos coletados na pesquisa. Observamos que em 2013 foram 34 laudos, enquanto em 2014 foram apresentou 44 casos. A tabela também mostra que os homens se mataram mais em $2014(86,24 \%)$, os solteiros com $54,5 \%$ e os casados $18,2 \%$. O método de morte mais utilizado nos 44 casos foi 0 enforcamento. No ano de 2013 os homens morreram mais, correspondendo a $91,2 \%$ da amostra entre 20 e 40 anos, entre esses suicídios os solteiros $(58,2 \%)$ morreram mais que os casados $(41,8 \%)$, utilizando em primeiro lugar o enforcamento $(82,4 \%)$ e em segundo lugar a arma de fogo $(17,6 \%)$. Outro dado apontado da Tabela 2 foi a média total de todos os anos da pesquisa e os dados sociodemográficos que indicou 0 número de suicídios de 5,6 para cada 100 mil habitantes.

\section{DISCUSSÃO}

$\mathrm{Na}$ presente pesquisa realizou-se uma análise dos dados obtidos nos prontuários do Instituto Médico Legal e também uma busca na literatura com a temática do suicídio. Foram também utilizados dados do Instituto Brasileiro de Geografia e Estatística2 $^{2}$ (IBGE, 2010), com o intuito de comparar o número da última pesquisa da população do estado de São Paulo, foi utilizado o número de habitantes divididos por sexo e estado civil. 0 porcentual e 0 número bruto de indivíduos da região do estado de São Paulo é composto por $51,34 \%(21.180,394)$ mulheres e 49,7 \% por pessoas casadas e $35,3 \%$ por solteiros. Assim, esses dados foram utilizados para fazer comparações com a amostra da pesquisa.

A hipótese levantada de que os homens cometem mais suicídios $(\mathrm{H} 1)$ foi baseada pelos dados da Organização Mundial da Saúde (OMS, 2014) e percebe-se que mesmo o estado de São Paulo possuindo

2. Organização pública responsável pelos levantamentos e gerenciamentos dos dados e estatísticas brasileiras. maior população feminina (IBGE, 2010), isso não interferiu no fato de que os homens apresentam maior índice de suicídio. A afirmação com poucos dados a corroborarem essa diferença de morte não está ligada ao número populacional, mas, aos aspectos psicológicos, sociais e biológicos que levam os homens a cometerem 0 ato do autoextermínio (Dutra, 2012).

Os resultados obtidos neste estudo indicaram maior prevalência de morte do sexo masculino, como já apareceu nas pesquisas de Parente, Soares, Araújo, Cavalcante e Monteiro (2007) em que $71,3 \%$ da população que cometeram suicídio foram os homens e Bezerra, Werneck, Almeida, Oliveira e Magalhães (2012) a qual mostrou que a população masculina se matou mais, uma vez que $64,4 \%$ dos suicídios contabilizados neste estudo eram de homens. De acordo com a OMS (2014) os homens se matam mais do que as mulheres, porque usam métodos mais efetivos e fatais como por exemplo, enforcamento e arma de fogo. Isso ocorre porque naturalmente 0 sexo masculino por ter dificuldades em demonstrar sentimentos em algumas situações, podem apresentar tendências emocionais mais agressivas, e por não conseguir lidar com emoções mais complexas podem ter atitudes mais severas ou agressivas. As mulheres tentam mais do que os homens, criando uma proporção de 3 tentativas para conseguir a morte, no entanto, os homens por utilizarem métodos mais letais se matam com mais frequência na primeira tentativa (Dutra, 2012).

No que se refere a idade, os dados nesta amostra não corroboram com a hipótese que pessoas idosas se matam mais $(\mathrm{H} 2)$ (OMS, coletados 2014). No presente estudo, a média de idade de suicídios dos homens e mulheres foram aproximadamente 43 anos, o que vai ao encontro do estudo de Viana, Zenkner, Sakae e Escobar (2008) os quais apresentaram que a região do Rio Grande 
do Sul, a média de idades variou de 40 a 45 anos. Pordeus, Cavalcanti, Vieira, Coriolano, et al. (2007) mencionaram em sua pesquisa no estado do Ceará que a idade prevalente com maior número de suicídios variou entre 40 a 49 anos, que foi a mesma faixa etária presente na pesquisa de Bezerra et al. (2012) realizada no estado do Rio de Janeiro.

Somente um estudo apareceu com a idade equivalente ao público da terceira idade como mostram Lovisi, Santos, Legay, Abelha e Valencia (2009) em que as mortes por suicídio ocorreram na faixa etária entre 59 e 70 anos. Lovisi, et al. (2009) fizeram um estudo que calculou as mortes por suicídio pelo Brasil. 0 estado de São Paulo apareceu em $3^{\circ}$ lugar com 4,9 suicídios a cada 100 mil habitantes, ficando atrás da região do Sul (10 lugar) com 9,4/100 mil habitantes e Centro-Oeste ( $2^{\circ}$ lugar) 7,4/100 mil habitantes. $O$ presente estudo encontrou resultados próximos aos dos autores citados no que diz respeito ao estado de São Paulo, que foram de 3,8 por cada 100 mil habitantes. Um aspecto importante a ser considerado em relação a este tema é o procedimento metodológico utilizado em cada uma das pesquisas. Viana, et al. (2008) comentam que a falta de identificação sociodemográfica em determinados prontuários dificulta a organização e o número exato de suicídios ocorridos em algumas regiões, podendo contribuir para diferenças metodológicas entre pesquisas com a mesma temática.

Outro dado sociodemográfico avaliado na pesquisa foi o estado civil, neste caso os solteiros correspondem a $43,4 \%$ dos suicídios. Comparado com o estudo de Baptista, et al. (2012), os solteiros também apresentaram maior índice de mortes com $38,2 \%$ da amostra total. Parente, Soares, Araújo, Cavalcante e Monteiro (2007) pesquisaram na região Nordeste do Brasil taxas de suicídio e avaliaram números maiores de suicídios nos solteiros, correspondendo a $67,6 \%$. Segundo os dados do IBGE (2010), a população geral do estado de São Paulo é formada em sua maioria por casados, porém, os solteiros se matam mais. Segundo Dutra (2012) Podese supor que os solteiros, não tendo vínculos sentimentais como por exemplo laços amorosos, acabam sendo mais vulneráveis a abalos emocionais, interferindo no seu repertório social.

Contrapondo com os números das pesquisas nomeando os solteiros como 0 estado civil mais propício a executar 0 ato do autoextermínio, outros estudos ficaram evidentes os casados como participantes em maior número de mortes. Como comentam Macente e Zandonade (2011), o número de suicídio dos sujeitos casados $(38,82 \%)$ foi superior, no entanto, com uma diferença mínima em relação aos indivíduos solteiros $(38,16 \%)$. Viana, Zenkner, Sakae e Escobar (2008) também encontraram um resultado semelhante, em que o número de casados $(28,6 \%)$ ficou bem próximo dos solteiros (26,5\%). Resultados similares foram identificados no presente trabalho, em que 0 suicídio foi mais frequente nos solteiros $(43,4 \%)$, seguido dos casados $(37,7 \%)$, indo ao encontro da literatura.

Por fim, os métodos mais utilizados no momento do suicídio, segundo os resultados do presente trabalho demonstraram o enforcamento e a arma de fogo como os métodos mais usados, o que corrobora a hipótese apresentada anteriormente, mostrando que 0 enforcamento apareceu em primeiro lugar. Viana, et al. (2008) mostraram em seu estudo que $68,4 \%$ de indivíduos se enforcaram. Pordeus, Cavalcanti, Vieira, Coriolano, Osório, Ponte e Barroso (2007) em uma pesquisa feita no Ceará também confirmaram que 0 enforcamento com $32,6 \%$ dos resultados finais foi o método mais efetivo para extinguir a própria vida. $\mathrm{A}$ morte com arma de fogo foi colocada em segundo lugar nos métodos fatais. Baptista, et al. (2005) e Baptista, et al. (2012), obtiveram resultados parecidos no caso de 
uso de arma de fogo, em que $13,8 \%$ e $18,8 \%$ utilizaram a arma de fogo respectivamente, confirmando com os dados da presente pesquisa.

A partir das discussões supracitadas percebe-se como 0 suicídio pode ser considerado um problema de saúde pública no Brasil, segundo a OMS (2014). Por isso é de suma importância o profissional da saúde conhecer mais sobre as questões que rondam 0 ato do suicídio, para conseguir fazer as intervenções necessárias com os indivíduos que tentaram executar 0 ato ou mesmo os familiares que passam por um abalo emocional (Dutra, 2012).

\section{CONCLUSÕES}

O presente estudo teve como objetivo inicial realizar uma revisão integrativa de estudos sobre epidemiologia de suicídios entre os anos de 2004 e 2014 e, em um segundo momento fazer uma análise descritiva das mortes ocasionadas por suicídio na região da cidade de Jundiaí, que engloba outros nove municípios, tendo como foco documental os laudos de um Instituto Médico Legal (IML). Outros estudos visando a análise epidemiológica e de levantamento contribuíram para um mapeamento especifica. A pesquisa também apontou algumas divergências comparadas com outros estudos, comparando-se os dados sociodemográficos, como por exemplo a idade, sexo e estado civil, que variam por estado pesquisado ou mesmo a cultura de cada população avaliada.

Algumas limitações foram percebidas no estudo, como na etapa da coleta de dados, onde informações pertinentes não foram encontradas com clareza, como por exemplo os municípios específicos onde foi realizado 0 ato do suicídio, doenças que poderiam estar ligadas com a morte, ou mesmo se 0 indivíduo tentou outras vezes se matar. Algumas dessas informações ajudariam muito na investigação sobre os comportamentos que estão diretamente ligados ao suicida, além disso, a coleta de dados não foi realizada por pesquisadores. Assim, não foi possível conferir erros na tabulação, sendo essas realizadas de forma manual ou mesmo a pesquisa dos laudos no sistema informatizado, o que pode ter aumentado a margem de erros na contagem manual e ter diminuído a média de notificações das mortes por suicídio.

Sugere-se que no futuro outras pesquisas com a metodologia de levantamento sejam realizadas, mas não só com dados sociodemográficos de prontuários de suicidas, mas também pesquisas com um foco qualitativo com os familiares de indivíduos que cometeram 0 ato do autoextermínio, para observar 0 fenômeno do impacto do suicídio na família e sociedade. Por fim, outra sugestão relevante seria replicar a pesquisa daqui cinco anos para mensurar se a média de suicídios da região teve alteração. 
REFERÊNCIAS

Baptista, M. N. (2004). Suicídio e depressão: atualizações. Rio de Janeiro: Guanabara Koogan.

*Baptista, M. N., Carneiro, A. M., Gomes, J. O., \& Cardoso, H. F. (2012). Análise Epidemiológica do Suicídio em duas Regiões do Estado de São Paulo entre 2004 e 2008. Psicologia em Pesquisa, 01, pp. 2-12.

*Baptista, M. N. \& Borges, A. (2005). Suicídio: aspectos epidemiológicos em Limeira e adjacências no período de 1998 a 2002. Estudos de Psicologia, 22(4). Campinas- SP.

Bertolote, J. M. (2012). O suicídio e sua prevenção. São Paulo, SP: Ed. Unesp.

Botega, N.J. (2015). Crise suicida: avaliação e manejo. Porto Alegre: Artmed.

*Bezerra, J. G. F., Werneck, G. L., Almeida, R. L., Oliveira, M. I., \& Magalhães, F. B. (2012). Estudo ecológico sobre os possíveis determinantes socioeconômicos, demográficos e fi siográficos do suicídio no Estado do Rio de Janeiro, Brasil, 1998-2002. Cad. Saúde Pública, 5, pp. 833-844.

Botega, N. J. (2014). Comportamento suicida: epidemiologia. Psicologia USP, 23(3), 231-236.

Correa, H., \& Barrero, S. P. (2006). O suicídio ao longo dos tempos. São Paulo, Atheneu.

DATASUS. (2014). Retirado 15 de Agosto de 2015, de http://datasus.saude.gov.br/nucleosregionais/minas-gerais/noticias-minasgerais.

Dutra, E. (2012). Suicidio de universitarios: o vazio existencial de jovens na. Estudos e Pesquisas em Psicologia, 12(3), pp. 924-937.

Hawton, K., Comabella, C. C., Haw, C. \& Saunders, K. (2013). Risk factors for suicide in individuals with depression: $A$ systematic review. Journal of affective, 147, pp. 17-28.

IBGE. (2014). Instituto Brasileiro de Geografia e Estatística. Retirado 12 de
Outubro de 2015, de

http://www.ibge.gov.br/home/estatistica /populacao/censo2010/default.shtm.

*Lovisi, G. M., Santos, S. A., Legay, L., Abelha, L., \& Valencia, E. (2009). Análise epidemiológica do suicídio no Brasil entre 1980 e 2006. Ver. Bras. Psiquiatr. pp. 86-94

*Macente, L. B., \& Zandonade, E. (2012). Spatial distribution of suicide incidence rates in municipalities in the state of Espírito Santo (Brazil), 2003-2007: spatial analysis to identify risk areas. Rev Bras Psiquiatr. 34, pp. 261-269.

Miret, M., Ayuso-Mateos, J. C., SanchezMoreno, J. \& Vieta, E. (2013). Depressive disorders and suicide: epidemiology, risk factors and burden. Neuroscience and Biobehavioral Reviews. 37, pp. 2372-2374

Ministério da Saúde (2009). Prevenção do suicídio: manual dirigido a profissionais da saúde da atenção básica. Brasília. Retirado 21 de Setembro de 2015, em HYPERLINK

"http://portal.saude.gov.br/portal/arquiv os/pdf/manu_prevencao240111.pdf"

Moreira, L. C., \& Bastos, P. R. (2015). Prevalência e fatores associados à ideação suicida na adolescência: revisão de literatura. Revista Quadrimestral da Associação Brasileira de Psicologia Escolar e Educacional, 19(3), 445-453.

Organização Mundial de Saúde - OMS. (2000). Classificação de Transtornos Mentais e de Comportamento da CID10: descrições clínicas e diretrizes diagnósticas. Porto Alegre: Artes Médicas.

Organização Mundial de Saúde (2014). Relatório sobre a Saúde Mental no Mundo. Genebra: OMS.

*Parente, A. d., Soares, R. d., Araújo, A. R., Cavalcante, I. S., \& Monteiro, C. F. (2007). Caracterização dos casos de suicídio em uma capital do Nordeste Brasileiro. Revista Brasileira de Enfermagem, 4, pp. 377-381. 
*Pordeus, J. M. A., Cavalcanti, L. P. G., Vieira, L. J. S, Coriolano, L. S., Osório, M. M, Ponte, M. S. R., \& Barroso, S. M. C. (2007). Tentativas e óbitos por suicídio no município de Independência, Ceará, Brasil. Ciência \& Saúde Coletiva, 5, pp. 1731-1740.

Suicídio: informando para prevenir / Associação Brasileira de Psiquiatria (2014). Comissão de Estudos e Prevenção de Suicídio. Brasília: CFM/ABP.

Viana, G. N., Zenkner, F. M., Sakae, T. M. \& Escobar, B. T. (2008). Prevalência de suicídio no Sul do Brasil, 2001-2005. J. Bras. Psiquiatr. 57(1), 38-43. 